\title{
Chinese Painting Elements in the Design of Custom Clothing
}

\author{
Liang Jun, Su Xin miao \\ Art Institute of Northeast China Electric Power University, Jilin
}

\begin{abstract}
Chinese painting as one of China's ancient culture and cultural heritage, with its style and artistic conception of deliberately deeply attracted the attention of international circles, and clothing as part of our daily life indispensable items, it not only represents our external kind of respect, but also a reflection of the personality connotation. This paper focuses on Chinese painting application status and characteristics of the elements in the custom apparel design is briefly summarized, and application design elements of Chinese painting made some resolution.
\end{abstract}

Keywords — Chinese painting elements, custom, costume design, aesthetic, value

\section{中国画元素在定制服装设计中的应用}

\author{
梁军宿金鍂炎 \\ 东北电力大学艺术学院, 吉林, 吉林, 中国
}

摘 要 中国画文化作为中国古老的传承文化之一, 以其风格的特意性和意境深远性深深的吸引了国际各界的关注, 而服装作为 我们日常生活中必不可缺的物品, 它不仅表示我们对外的一种尊重, 也是人格内涵的一种体现。本文主要对中国画元素在定制服装设 计中的应用现状和特征进行了简要概括，并对中国画元素的应用设计进行了一些解析。

关键词中国画元素，定制，服装设计，审美，价值

\section{1. 中国画的审美对定制服装设计的特征表现}

中国画的精髓就在于绘画手法在画中表达的内涵, 因 此，在定制服装设计中的中国画元素也一定会在这两个方 面有所渗透, 具体体现在笔墨、构图以及色彩三个方面的 中国画理念设计表。

笔墨是最能直接体现中国话元素的一种技法, 在定制 服装设计上通过中国画笔墨的渲染来扩大服装理念的张 力, 让服装能够快速吸引顾客的目光, 从而表达一种丰富 的文化底蕴。

构图是中国画的中心，作者通过描绘不同的构图给不 同的服装进行搭配, 从而让定制服装能够具有更好的视觉 效果, 也为服装设计者提供一定的灵感, 作者通过不同的 构图来表达不同的意境。

色彩可以为定制服装设计作品中的中国画注入灵魂, 能够让作品的美感更加突出, 色彩的应用是定制服装设计 的重要因素, 不仅能够体现出服装的张力也可以体现出服 装的神韵。

\section{2. 中国画审美家价值对定制服装设计影响}

\section{1 灵感来源及意境设计}

由于现代人们对于服装的追求已经从以前保暖蔽体的 基本要求变为对美感的体会和精神上的享受, 而中国画元 素在服装设计上的搭配则恰好可以让服装更加贴近人心， 在满足群众对美感的追求的同时也是对我国中华传统文化 的一种宣传。

定制服装设计师首先就要对服装的主题有一个大概的 认识, 中国画元素以其风格多元、色彩多变、意境深远、 线条富有节律感等多方面的特点给设计师创造了大量的灵 感, 从而让设计师能够设计出具有艺术魅力的优秀作品, 让服装的设计更好的满足顾客的需求。

\section{2 轮廓与图案色彩搭配设计}

定制服装的轮廓与图案设计在加入中国画元素后使得 衣服的风格变得更加飘逸空灵, 设计师在服装设计时很好 
地体现了服装的那种层次美, 展示了中国文化的博大内涵, 给人一种自然清新、大气勃发的视觉感受。

中国画元素在轮廓的设计上打破了传统衣服的那种单 一感, 让衣服出现一定的层次, 显得更加飘逸出尘, 而在 图案和色彩的搭配上, 中国画以其笔墨的挥酒和强烈的色 彩差和留白来消除客户的审美疲劳, 让客户感受到一种轻 松自在的感觉, 无形中接受了设计师的设计理念和风格, 从而满足了客户对定制服装的设计需求。

\section{3. 中国画元素在定制服装设计中的应用}

\section{1 装饰部位应用}

定制服装设计作品的好坏评价不仅仅是以服装的轮廓 构造和色彩搭配来衡量的, 在服装的修饰以及装饰部位的 选择都能在很大程度上为服装增彩, 因此, 在定制服装的 肩部、领口、腰部、衣襟、下摆等部位进行中国画元素的 适当装饰, 不仅能够在细节上体现作品的风格特色, 甚至 会产生很多意外的惊艳装饰效果, 从而给人耳目一新的感 觉。

例如在 08 年罗峥发布的《人面桃花》作品系列, 运用 中国画桃花的图案给服装进行以印花、亮片、钉珠以及刺 绣等手法的装饰, 使得中国画桃花元素显得十分立体, 给 人一种强烈的直观视觉冲击, 让顾客对作品的注意力充分 集中到作品上去, 装饰部位的极大地体现了作者在服装设 计上的特点, 同时也使服装的设计主题更加突显, 极具中 国文化的韵律之美。

通过服装的剪裁以及装饰的布局架设, 能够使人们在 感受到作品所想要传达的内容的同时, 又不会感觉过于疲 劳。

\section{2 面料选择应用}

中国画元素在定制服装设计的面料选择上的应用也随 着面料的种类的不同有着不同的结合方式, 当与如丝绸、 纺纱等轻薄面料进行搭配时, 要注重表现出服饰的轻灵、 飘渺之美, 可以利用工笔花鸟画作为修饰, 给人一种精致、 淡雅的感觉。

当与秧苗毡、涤纶等厚重面料进行搭配时, 可以选择 以黑白色调为基础的山水画系列与服装进行整合渲染, 这 种画面线条粗狂的画风能够让定制服装的整体感和存在感 加强, 给人一种厚重、高贵的感觉。而那些如反光的特殊 面料就要突出服装的工艺效果,

如 0scar De La Renta 品牌在这种面料设计时创新使用 了数字喷墨印花墨竹图案, 这种数字喷墨的印花技术在很 大程度上加强了对中国画元素的表现, 给人强烈的反光视
觉冲击, 从整体上展示了中国文化的古韵内涵, 从而让服 装设计特色更加突显。

\section{3 款式搭配应用}

在款式搭配上的中国画元素应用, 定制服装设计要从 宽松和合体两种角度进行思考设计。

宽松的服装设计往往用料较多, 所以可以采用整体画 面印刷的方式, 中国画因其构图、笔墨、色彩等多种原因 本身就具有很高的审美价值, 将整幅中国画运用到定制服 饰上能够体现服装的整体美感。

而合体的服装则要保证中国画元素的意境完全被诠释 出来, 例如在 NE-TIGER 的 13 年新品发布会上展示的作品, 运用中国画梅花进行创新使用的局部褶皱重点装饰, 让梅 花这种具有零散装饰效果的中国画元素特色在局部褶皱中 表现出来, 突显了服装的三维立体感, 让服装看上去也更 加的夺目, 使顾客对定制服装的设计意境和主题一目了然。

\section{4. 结语}

随着中国的日益强盛, 中国文化也渐渐受到世界范围 的广泛关注, 中国画元素在各行各业上的应用也越来越普 遍。

将中国画元素运用到定制服装设计上, 能够为服装赋 予生命力和感染力, 提高服装作品的古韵内涵, 这既满足 了人们对于服装美感的需求, 也让中国画文化得到了传播。 将中国画元素与定制服装设计理念相融合, 充分发掘出我 国民族文化的美感与内涵, 运用独特的艺术手法进行创新 和修饰，让服装的色彩、纹样和肌理的中国画审美价值充 分展示出来, 从而不断将中国文化不断发扬光大。

\section{参考文献(References)}

[1] Du Liwei. On the combination of traditional culture and modern fashion design application. Modern Reading (Education Edition) 2012 (24)

[2] Dan. On application of traditional Chinese elements in fashion design. Harbin Institute. 2012 (12)

[3] Yu Weihua. Analysis of innovative applications in the traditional elements of Chinese clothing. Beauty and the times (the) 2010 (10)

[4] From Wei, Zhou Liying. Talks costume design and postmodern art fusion. Art and Design (theoretical) 2010 (03)

[5] Hui Chin. Discussion on building deconstruction strategy in fashion design application. Art and Design (theory). 2011 (06)

[6] Cynthia Tseng. 15 anniversary of the British Fashion Zhouzuo Yu GFW graduate design excellence. color in 2006 (09)

[7] Huyue Qin, while Xiaofang. Scale modern women talk about innovative design. Art Grand 2009 (08) 
[8] Chen Bin. Psychological impact of visual color costume design. Popular color. 2010 (11)

[9] Jin Ying, Lin Jian. Apparel industry in the current wave of cross-border exploration. Art and Design (theoretical) 2011 (08)

[10] Hanjiang red. Designers and fashion design as a commodity. Zhejiang Arts and Crafts in 2004 (02)

[11] Shu Yuan costume design event decorate creative life -.
Remember 2008 Shanghai International Fashion Culture Festival Shanghai Glance 2008 (05)

[12] Tao Yi thought process about fashion design decoration. 2006 (02)

[13] Liu Zhouhai senior designer. Talking about fashion design and creative thinking and physical methods. Fine Grand. 2008 (10) 